\title{
Magnetization reversal and confinement effects across the metamagnetic phase transition in mesoscale FeRh structures
}

ARREGI URIBEETXEBARRIA, J.; HORKÝ, M.; FABIANOVÁ, K.; TOLLEY, R.; FULLERTON, E.; UHLÍR̆, V.

Journal of Physics $D$ - Applied Physics

2018, vol. 51, iss. 10 , pp. 1-12

ISSN : 1361-6463

DOl: http://dx.doi.org/10.1088/1361-6463/aaaa5a

Accepted manuscript

(C) 2018 IOP Publishing Ltd 


\title{
Magnetization reversal and confinement effects
}

\section{across the metamagnetic phase transition in mesoscale FeRh structures}

\author{
Jon Ander Arregi ${ }^{1}$, Michal Horký ${ }^{1}$, Kateřina Fabianová ${ }^{2}$, Robert Tolley ${ }^{3}$, Eric E. Fullerton ${ }^{3}$, \\ and Vojtěch Uhlír̆ ${ }^{1,2}$ \\ ${ }^{1}$ CEITEC BUT, Brno University of Technology, Purkyňova 123, 61200 Brno, Czech Republic \\ ${ }^{2}$ Institute of Physical Engineering, Brno University of Technology, Technická 2, 61669 Brno, Czech Republic \\ ${ }^{3}$ Center for Memory and Recording Research, University of California San Diego, La Jolla, \\ California 92093-0401, USA \\ *E-mail: ja.arregi@,ceitec.vutbr.cz, vojtech.uhlir@ceitec.vutbr.cz \\ J. Phys. D: Appl. Phys. 51, 105001 (2018) - Published on 15th February 2018 \\ https://doi.org/10.1088/1361-6463/aaaa5a
}

\begin{abstract}
The effects of mesoscale confinement on the metamagnetic behavior of lithographically patterned FeRh structures are investigated via Kerr microscopy. Combining the temperatureand field-dependent magnetization reversal of individual sub-micron FeRh structures provides specific phase-transition characteristics of single mesoscale objects. Relaxation of the epitaxial strain caused by patterning lowers the metamagnetic phase transition temperature by more than $15 \mathrm{~K}$ upon confining FeRh films below $500 \mathrm{~nm}$ in one lateral dimension. We also observe that the phase transition becomes highly asymmetric when comparing the cooling and heating cycles for 300-nm-wide FeRh structures. The investigation of FeRh under lateral confinement provides an interesting platform to explore emergent metamagnetic phenomena arising from the interplay of the structural, magnetic and electronic degrees of freedom at the mesoscopic length scale.
\end{abstract}




\section{INTRODUCTION}

The chemically ordered (CsCl-type) FeRh-alloy close to equiatomic composition undergoes a first-order metamagnetic phase transition from antiferromagnetic (AF) to ferromagnetic (FM) order upon warming above room temperature $(\sim 370 \mathrm{~K})[1-3]$. In the low temperature phase, FeRh is a G-type antiferromagnet where Fe atoms carry a moment of $\pm 3.3 \mu_{\mathrm{B}}$, while $\mathrm{Rh}$ atoms possess a negligible magnetic moment. On the other hand, the local $\mathrm{Fe}$ and $\mathrm{Rh}$ moments in the FM phase are ferromagnetically coupled, amounting to $3.2 \mu_{\mathrm{B}}$ and $0.9 \mu_{\mathrm{B}}$, respectively $[4,5]$ (see Figure 1). The AF-FM transition is accompanied by an isotropic lattice expansion $(\sim 0.6 \%)$ and a reduction in resistivity $(\sim 50 \%)$, possessing coupled structural, electronic and magnetic order parameters [6, 7]. For films the transition features a thermal hysteresis of around $10 \mathrm{~K}$ between the cooling and heating cycles and it can be controlled via multiple external driving forces, such as temperature, magnetic field [8], strain [9-11], electrical currents [12-14] or ultrafast optical pulses [15-17]. The versatility to trigger and control the transition, as well as the associated changes in magnetization, magnetoresistance and entropy make FeRh an interesting candidate for incorporation into novel magnetic recording [18] and spintronic [19] devices, as well as for active components of magnetic refrigerators [11, 20, 21]. Among other metallic materials that exhibit a similar AF to FM phase transition (such as doped $\mathrm{CeFe}_{2}$ compounds [22]) the feature at which FeRh is prominent consists in that the phase transition occurs near room temperature.

In FeRh thin films, the transition broadens due to disorder and heterogeneity, caused by elements such as local structure or stoichiometry variations. As different parts of the sample possess slightly different transition temperatures, AF and FM domains coexist while the sample progresses through the phase transformation, leading to the smearing out of the sharp transition. Along these lines, Ohtani and Hatakeyama discuss the role of crystallographic defects as well as $\mathrm{Rh}$ - and Fe-rich precipitates in the CsCl-type FeRh matrix, which cause the AF phase to be locally unstable $[23,24]$ and thus leading to an AF-to-FM transition that is more cooperative than the opposite FM-to-AF transformation [24, 25]. In case of highly ordered epitaxial thin films, some studies have observed modest signatures of asymmetry in the phase transition when comparing the cooling and heating cycles, in terms of phase separation and domain nucleation kinetics $[26,27]$.

In contrast to the vast literature addressing properties of FeRh thin films, there are only few reports on patterned FeRh [12-14, 28, 29]. Uhlír et al. [29] reported that patterning FeRh 
films into stripes of mesoscale dimensions results in a dramatic enhancement of the phase transition asymmetry. Specifically, it was found that when tracking the resistance of submicron-wide FeRh wires, a broad transition was observed upon heating, while a collective response featuring a transition in few abrupt jumps as well as suppression of the phaseseparated state appeared during cooling. The threshold stripe width at which this behavior emerges corresponds to the characteristic size of AF and FM phase domains during coexistence in thin films $(\sim 0.5 \mu \mathrm{m})$ [27]. The origin of this asymmetry was attributed to the fact that while long-range ferromagnetic order persists through regions of disorder, long-range antiferromagnetic order is easily disrupted by defects [29], for instance, crystallographic grain boundaries which constrain the AF domain size [30]. Thus, the exact character of the transition asymmetry in FeRh appears to depend on the combination of the structural and magnetic degrees of freedom, with the distinct effect of defects on the AF and FM order playing an important role at the mesoscale.

Further studies of meso- and nano-scale structures are needed in order to gain a better understanding of confinement effects in FeRh, which could ultimately lead to the discovery of emergent phenomena that are interesting for novel spintronic devices. In particular, the recovery of the abrupt phase transition upon mesoscale confinement of thin films could be beneficial for achieving a low-energy activation of the phase transition in FeRh. In this work, we study the metamagnetic behavior and magnetization reversal properties of mesoscale FeRh structures. The FeRh patterns investigated here possess square or rectangular geometry with characteristic sizes ranging from $0.3 \mu \mathrm{m}$ to $20 \mu \mathrm{m}$. The structures were imaged by wide-field magneto-optical Kerr effect microscopy, which in spite of being restricted by the optical resolution limit, allows us to examine an array of several FeRh structures simultaneously. In fact, magneto-optics proved to be suitable for the study of nanostructures, both via wide-field microscopy [31,32] and focused laser approaches [33-35]. While spatially resolved reflectivity and transmittance measurements have been recently employed for the study of the phase transition in FeRh films [36], Kerr microscopy offers the additional advantage of being sensitive to magnetization processes. This is useful to establish, for instance, how the shapeinduced magnetic anisotropy affects the magnetization reversal properties of FeRh. By tracking the temperature-dependent variation of the Kerr contrast across the transition, we systematically characterize the metamagnetic behavior of individual FeRh mesoscale structures. We observe that (i) structures confined to lateral sizes below one micron undergo a substantial variation of their transition temperature as a result of a strain relaxation process 
resulting from patterning, and that (ii) the asymmetric phase transition, originally observed using resistivity measurements, is reproduced in magnetization for the narrowest FeRh rectangles. Our results contribute to understanding of the coexistence state of the AF and FM phases upon mesoscale confinement, which constitutes an interesting platform for potential memory or logic devices $[37,38]$.

\section{EXPERIMENTAL DETAILS}

For the present study, 50-nm-thick epitaxial FeRh films were deposited onto single-crystal $\mathrm{MgO}$ (001) substrates from an equiatomic FeRh target, using a high vacuum chamber with a base pressure of $10^{-8}$ Torr. The substrates were preheated to $450^{\circ} \mathrm{C}$ prior to deposition and the same temperature was maintained during growth, which was done at an Ar pressure of $2.2 \times$ $10^{-3}$ Torr. Subsequently, the films were annealed at $800^{\circ} \mathrm{C}$ for 45 minutes in order to obtain the desired CsCl-type (B2 ordering) crystal structure, which leads to the presence of the AF to FM phase transition at $\sim 370 \mathrm{~K}[8,39-41]$. A 2-nm-thick Pt protective capping layer was deposited on the FeRh films once they were cooled down to a temperature below $100^{\circ} \mathrm{C}$. X-ray diffraction measurements confirmed the out-of-plane FeRh (001) preferential orientation as well as the achievement of in-plane epitaxy of the films with the $\mathrm{MgO}$ substrate, showing the same high texture level reported earlier [29]. To demonstrate the metamagnetic phase transition in our FeRh layers, Figure 2(a) displays the saturation magnetization vs. temperature loop for the film we patterned for this work, measured via vibrating sample magnetometry at an in-plane applied field of $1 \mathrm{~T}$. The FeRh film shows a rather broad transition during both heating and cooling cycles $(\sim 40 \mathrm{~K})$, together with a very low residual ferromagnetic fraction $(\sim 2 \%)$ at $320 \mathrm{~K}$. The saturation magnetization of $1140 \times 10^{3} \mathrm{~A} / \mathrm{m}$ at $400 \mathrm{~K}$ is in good agreement with previous studies on FeRh films [8, 27, 39].

Mesoscale FeRh structures were fabricated from the source film by electron beam lithography using a negative resist (hydrogen silsesquioxane - HSQ) followed by ion etching in Ar plasma and cleaning the resist residuals by buffered oxide etch (hydrofluoric acid and ammonium fluoride). Figure 2(b) shows a scanning electron micrograph of FeRh rectangles and squares with lateral sizes between 0.3 and $20 \mu \mathrm{m}$. The chemical composition contrast of the FeRh structures and the $\mathrm{MgO}$ substrate was achieved by detecting back scattered electrons 
(SEM-BSE). Figure 2(c) shows a magnified region of the micrograph in Figure 2(b), displaying the good quality of $20-\mu \mathrm{m}$-long stripes and squares with sub-micron lateral sizes.

Magnetization properties of mesoscale FeRh structures were studied using a highresolution optical microscope with polarization analysis, by retrieving the magnetic domain contrast due to the magneto-optical Kerr effect. A fiber coupled light-emitting diode lamp providing a broad spectrum of wavelengths in the range of 450-700 $\mathrm{nm}$ was employed as the light source. The sample holder is equipped with a high-vacuum environment cryostat and an electromagnet, which allows performing Kerr microscopy at temperatures between 300-450 K with 1-K resolution and applied in-plane magnetic fields up to $150 \mathrm{mT}$. While special configurations of Kerr microscopy currently allow pushing the spatial resolution down to $\sim 0.2$ $\mu \mathrm{m}$, a resolution limit of around $0.5-1 \mu \mathrm{m}$ is achieved with the microscope utilized here using a $50 \times$ objective. In fact, the need for an optical window due to the implementation of a cryostat does not allow achieving the highest spatial resolution in the present case. For a comprehensive review, see Ref. 32. However, it has been recently demonstrated that despite the resolution limitation, Kerr microscopy enables measuring magnetic hysteresis loops with a remarkable signal-to-noise ratio even from individual nanostructures with sub-100-nm lateral dimensions [31].

Figure 2(d) exhibits a Kerr microscopy image retrieved at $400 \mathrm{~K}$ over a region with an area of $150 \times 50 \mu \mathrm{m}^{2}$, where a series of FeRh squares and rectangles shown by the SEM image in Figure 2(b) is contained. The Kerr micrograph is acquired at an applied field of $40 \mathrm{mT}$ after subtracting the background at zero field. The FeRh structures can be discerned from the $\mathrm{MgO}$ substrate by the brighter grayscale contrast due to the magneto-optical Kerr effect. For each of the patterns in Figure 2(d), we have defined a rectangular region of interest (ROI), indicated by red or blue for squares or rectangles, respectively. These are defined to enclose each mesoscale structure and leaving a lateral space of $\sim 1-2 \mu \mathrm{m}$ on the sides. We have encoded each ROI by using the identifiers " $\mathrm{S}$ " and "R" for squares and rectangles, as well as a number indicating its feature size in microns. Thus, for instance, "S20" refers to a square with a 20$\mu \mathrm{m}$-long side, while "R0.5" denotes a $20-\mu \mathrm{m}$-long and $0.5-\mu \mathrm{m}$-wide stripe [see Figure 2(d)]. An additional ROI with the identifier "BG" (background) is defined to obtain the Kerr signal originating from the etched region of the film, where only $\mathrm{MgO}$ remains.

Magnetization reversal in individual structures is studied by sweeping the applied magnetic field at a constant temperature and evaluating the grayscale variation corresponding 
to the magneto-optical signal inside each ROI. When investigating magnetization reversal across the metamagnetic phase transition, thermal effects cause mechanical drifts and refocusing is needed each time a different temperature is set. Thus, positioning of the depicted ROI map is corrected for each temperature step such that it matches the configuration established in Figure 2(d). For the experiments shown here, we have chosen the longitudinal Kerr geometry, in which the magnetic domain contrast varies according to the magnetization component in the vertical direction of the Kerr micrograph in Figure 2(d). The applied magnetic field was also defined along this direction, as illustrated by the arrow in the top right corner of Figure 2(d). The choice of this particular field orientation and Kerr sensitivity is based on the shape anisotropy of rectangular FeRh structures acquired upon patterning.

\section{RESULTS AND DISCUSSION}

\section{A. Magnetization reversal behavior in the FM phase}

The magnetization reversal of individual mesoscale structures was initially investigated in the fully FM phase. Figure 3(a) displays sequential snapshots of the region previously shown in Figure 2(d) during magnetization reversal at $400 \mathrm{~K}$, where the grayscale represents the magnetic domain contrast due to the longitudinal Kerr effect. For an applied field of $20 \mathrm{mT}$, the FeRh patterns show a brighter grayscale contrast than the background, indicating that they are uniformly magnetized. During the decreasing field branch, in the left panel of Figure 3(a), we observe domain nucleation occurring in the largest FeRh squares and rectangles at an applied field of $-10 \mathrm{mT}$, while the narrower FeRh rectangles still preserve a uniform magnetization state. At an applied field of $-12 \mathrm{mT}$, the few-micron-wide rectangular structures have generally reversed their magnetization, as shown by the darker grayscale perceived at their position, while many of the sub-micron rectangles still display a brighter domain contrast, i.e. they have not switched yet. At the last snapshot in the left panel of Figure 3(a), corresponding to $-20 \mathrm{mT}$, all discernible FeRh structures in the image appear to have reversed their magnetization, as concluded from the uniform, dark domain contrast exhibited by the FeRh structures. Snapshots corresponding to the increasing field branch are also shown in the right panel of Figure 3(a), where the equivalent magnetization reversal behavior follows in the inverse direction. Apparently, the coercive field increases with decreasing the structure size, which is the expected behavior as the number of nucleation sites for magnetization reversal is 
reduced [42]. It is worth noting that none of the sub-micron square structures is appreciable in the Kerr micrographs in Figure 3(a), while the domain contrast from the FeRh rectangles can be discerned, although substantially diminished, even for widths below one micron.

We confirm the qualitative conclusions extracted from domain observation in Figure 3(a) by quantitatively evaluating the longitudinal Kerr signal $I$ within the ROIs defined for each FeRh mesoscale structure. Magnetic hysteresis loops for the FeRh square S20 as well as the rectangles R10, R1 and R0.4 are exhibited in Figure 3(b). All hysteresis loops shown here display a high signal-to-noise ratio, even for the structure R0.4, which consist of a $20-\mu \mathrm{m}$-long and 0.4- $\mu \mathrm{m}$-wide rectangle. The specific applied field values for the snapshots shown in Figure 3(a) are designated with triangular markers on top of the hysteresis loops in Figure 3(b), together with the specific snapshot numbers adjacent to the marker. The hysteresis loop features in Figure 3(b) confirm the magnetization reversal properties we have deduced from the Kerr micrographs in Figure 3(a). For instance, the structures S20 and R10 undergo reversal via magnetization rotation and domain nucleation, as intermediate magnetization states are apparent near the coercive field value. Opposite to this, the narrower rectangular structures R1 and R0.4 are characterized by a square hysteresis loop and the absence of intermediate magnetization states, from which domain wall propagation can be identified as the main reversal mechanism.

In order to give further evidence that the FeRh structures are fully ferromagnetic at 400 $\mathrm{K}$, Figure 4(a) displays the magnetic domain contrast at $400 \mathrm{~K}$, which is obtained by subtracting the Kerr micrographs at opposite applied fields of $\pm 40 \mathrm{mT}$. Apparently the areas of the rectangles and squares with feature sizes of $1 \mu \mathrm{m}$ and above are completely defined by their magnetic domain contrast. In the top-right part of Figure 4(a) we recognize the magnetic domain contrast arising from the rectangles of sub-micron width, although the spatially resolved signal is manifestly smeared out due to the fact that we are reaching the resolution limit of the microscope. However, we are not able to retrieve any detectable magnetic domain contrast from the sub-micron squares located in the bottom-right part of Figure 4(a).

The detection limit for FeRh squares and rectangles at $400 \mathrm{~K}$ is quantified from the magnetic hysteresis loops. Figures 4(b)-(d) exhibit hysteresis loops for the squares S5, S1 and S0.8. While S5 displays a well-marked hysteresis loop, the variation of the Kerr signal $I$ arising from S1 upon sweeping the field is minimal, being completely lost for the S0.8 structure. This is in accordance with the magnetic resolution limit for Kerr microscopy under the conditions 
utilized here [32]. On the other hand, hysteresis loops with a relatively good signal-to-noise ratio are obtained for rectangles with widths down to $0.3 \mu \mathrm{m}$, which is the minimum size considered in this study. Figures 4(e)-(g) contain the field-dependent Kerr signal $I$ arising from the FeRh rectangles $\mathrm{R} 4, \mathrm{R} 0.7$ and $\mathrm{R} 0.3$, which all display square-shaped loops. As the relative scale of the Kerr signal for each of the plotted loops is kept, narrower rectangles demonstrate a corresponding reduction in the signal amplitude. The Kerr signal arising from the area of the sample denoted as BG, where no FeRh is present, is shown for comparison on top of the S5 data in Figure 4(b), displaying no substantial field-dependent variations.

The magnetic hysteresis loops of rectangular FeRh structures shown in Figures 3 and 4 exhibit a monotonic increase of the coercive field $\mu_{0} H_{c}$ upon reducing their width. The comprehensive coercive field dependence on the size of FeRh rectangles as well as squares down to $1 \mu \mathrm{m}$ is shown in Figure 4(h). While the $\mu_{0} H_{c}$ vs. size dependence does not exhibit any clear trend for FeRh squares, coercive field of rectangular structures increases from about $10 \mathrm{mT}$ to $27 \mathrm{mT}$ when the width is reduced from $10 \mu \mathrm{m}$ to $0.3 \mu \mathrm{m}$. Along the same lines, the inset in Figure 4(h) shows the $\mu_{0} H_{c}$ data for the FeRh rectangles as a function of the aspect ratio (AR), where a clear linear trend is observed. We notice that such an increase of the coercive field occurs in a very similar fashion in patterned magnetic materials with elongated shape, such as permalloy $[43,44]$ or polycrystalline Co [31], at least in the regime AR $>2$, which is our case here.

Such a coercivity increase with increasing the AR can be explained in terms of the uniaxial magnetic shape anisotropy acquired upon patterning FeRh into elongated structures, given that magnetostatic interactions become relevant during magnetization reversal. Other effects influencing the coercivity increase are not expected to play an important role: (i) the in-plane four-fold magnetocrystalline anisotropy of FeRh thin films should be negligible [39], and (ii) dipolar magnetic fields arising from neighboring patterns are insignificant as well, given that the structures are separated by a minimum distance of $3 \mu \mathrm{m}$.

\section{B. Magnetization reversal behavior across the metamagnetic phase transition}

After characterizing the magnetization reversal and the Kerr signal level of mesoscale FeRh structures in the FM phase, we focus on the magnetization reversal across the AF-FM phase transition. In order to study the effect of confinement via Kerr microscopy, we focus on 
rectangular structures, in which one lateral dimension is substantially decreased while still keeping a reasonable signal-to-noise ratio. Figure 5(a) shows exemplary hysteresis loops for the rectangles R4, R1 and R0.3, acquired at different temperatures during the cooling cycle. For the loops shown here, we have scaled the Kerr signal variation $\Delta I$ for each structure size to the normalized Kerr signal of magnetization saturation at $390 \mathrm{~K}$. In such a way, the evolution of the Kerr signal amplitude can be followed for subsequent temperatures. For instance, we observe that the data at $378 \mathrm{~K}$ show a slight reduction of the Kerr signal for the structure R4, while R1 and R0.3 keep the same signal amplitude as that retrieved at $390 \mathrm{~K}$ upon sweeping the field. As the temperature is further lowered down, at $366 \mathrm{~K}$ the signal reduction for $\mathrm{R} 4$ is more evident, while only a slight reduction can be observed for R1. In contrast, R0.3 still shows the same Kerr contrast amplitude in magnetic saturation as the loop retrieved at $390 \mathrm{~K}$. In fact, a similar amplitude level is kept for R0.3 until the temperature drops below $360 \mathrm{~K}$ [see Figure $5(\mathrm{a})]$.

This indicates that the rectangular structures of different sizes undergo the FM-to-AF transition at different temperatures, with the transition generally starting at lower temperatures for narrower rectangles. The additional hysteresis loops in Figure 5(a) display how the Kerr contrast continues diminishing upon decreasing the temperature to the point that any hysteresis loop becomes undetectable at a temperature of $340 \mathrm{~K}$. Magnetic hysteresis loops for the same FeRh structures during the heating cycle, showing the inverse path of the transition from the AF-to-FM phase, are shown in Figure 5(b). At $340 \mathrm{~K}$, no detectable Kerr contrast is found upon sweeping the applied magnetic field and the magnetic hysteresis loop first appears once a temperature of $360 \mathrm{~K}$ is reached. The Kerr contrast amplitude seems to grow earlier for the R0.3 rectangle, following the trend found for cooling in which the narrower rectangles undergo the phase transition at lower temperatures.

Characterization of the phase transition features of each individual structure was done by quantifying the temperature-dependent Kerr contrast $\Delta I$ in magnetic saturation, which is plotted in Figures 6(a)-(c) for the structures R4, R1 and R0.3. The value $\Delta I$ has been scaled here with respect to the maximum Kerr contrast $\Delta I_{\max }$ for each structure, such that a $\Delta I / \Delta I_{\max }$ value of 0 or 1 would indicate a completely AF or FM phase, respectively. The data in Figure 6 allows us to directly follow the magnetization vs. temperature behavior for each mesoscale FeRh rectangle in great detail during both the cooling and heating cycles. Metamagnetic behavior in mesoscale FeRh structures has only been studied, so far, via transport 
measurements [12-14, 29] or resonant scattering of polarized x-rays [28]. In Figures 6(a)-(c), we have also represented the magnetization vs. temperature hysteresis loop of the source FeRh film (dashed lines) together with the Kerr microscopy data, from which it is evident that the phase transition of the film is significantly broader when compared to mesoscale structures. This results from the variations of the transition temperature within the film, as we average a large area $\left(5 \times 5 \mathrm{~mm}^{2}\right)$ with slightly varying stoichiometry or density of structural defects. In contrast, when confining the film into structures with an area of $\sim 1-100 \mu \mathrm{m}^{2}$, we can assume that the distribution of locally varying structural properties narrows down considerably, such that a sharper phase transition occurs generally in patterned FeRh structures.

Furthermore, we can find a number of differences in the metamagnetic behavior of $\mathrm{FeRh}$ rectangles with varying widths. For instance, a lower transition temperature is observed for narrower wires both during the cooling and heating cycles, in line with the conclusions we made from the observations in Figure 5. We associate this noticeable reduction of the transition temperature with a strain relaxation process introduced by patterning. It is well known that $\mathrm{FeRh}$ thin films grown on $\mathrm{MgO}$ feature a compressive in-plane strain, causing an increase of the transition temperature when compared to unstrained, bulk FeRh [8, 39]. Upon patterning, an increase of the surface-to-volume ratio occurs in the FeRh structure, allowing the lattice to relax in the lateral directions and bringing an associated decrease of the transition temperature with it. While the transition temperature dependence of FeRh on its strain state is well known, a shift of its value upon patterning of strained films into mesoscale structures had not been observed so far.

In order to study quantitatively the variation of the transition temperature dependent on the FeRh rectangle size, we have fitted the temperature-dependent Kerr contrast to the following sigmoid function

$$
\left(\frac{\Delta I}{\Delta I_{\max }}\right)_{i}=A\left[\frac{1}{2}+\frac{1}{\pi} \arctan \left(\pi \frac{T-T_{i}}{w_{i}}\right)\right]+B
$$

where the index $i$ refers to either the FM-AF (cooling) or AF-FM (heating) transition, with $T_{i}$ being the transition temperature fit parameter. Additionally, we introduce $w_{i}$ as a parameter related to the transition width, while $A$ and $B$ are fit parameters that slightly deviate from 1 and 0 , respectively, in order to facilitate the fitting process. From the fits to the data, we have 
obtained transition temperatures for the structures R4, R1 and R0.3 during cooling, which are $366.5 \pm 0.3 \mathrm{~K}, 362.6 \pm 0.5 \mathrm{~K}$ and $353.7 \pm 0.4 \mathrm{~K}$, respectively. The transition temperatures during heating are $386.6 \pm 0.6 \mathrm{~K}, 381.7 \pm 1.6 \mathrm{~K}$ and $369.8 \pm 1.0 \mathrm{~K}$ (see Figure 6).

Apart from the shift in the transition temperature, we also observe differences in the transition character. While the transition during heating occurs gradually for all the structures in Figure 6, a different behavior is seen during cooling. In particular, the structures R4 and R1 display a broad transition during cooling, suggesting that AF and FM domains coexist over a range of around $20 \mathrm{~K}$ [Figures 6(a) and (b)]. However, the transition during cooling becomes very sharp for R0.3 [Figure 6(c)], happening in just few abrupt jumps. While our temperature resolution of $2 \mathrm{~K}$ does not allow checking for all intermediate states across the transition, the data in Figure 6(c) suggests that the coexistence of AF and FM phases is largely suppressed and limited to a temperature span of a few $\mathrm{K}$. This asymmetric behavior is very similar to the one we found earlier while tracking the resistance of sub-micron FeRh wires across the metamagnetic transition [29]. These current results confirm that the previous findings were not related to the current used to probe the transition. It was argued that this asymmetry arises from the different role of AF and FM order at the mesoscale. In the low-temperature phase, agents such as crystallographic defects or slight stoichiometry variations compromise the long-range AF order, which is known to be shorter or equal to the measured structural coherence length $[30,45]$. Thus, when the system progresses through the AF-FM transition, uncorrelated AF portions undergo the phase change to the FM state at different stages, resulting in a gradual transition. Opposite to this, the FM order is not nearly as susceptible to defects such as crystallographic disorder and can persist even across crystallographic grain boundaries. This causes that when FeRh is confined into mesoscopic structures, the FM interactions are strong enough to stabilize the system and favor the collective transition over mesoscopic areas, hence suppressing the phase coexistence and recovering the abrupt nature of the first-order phase transition. Our Kerr microscopy results confirm that the asymmetry in mesoscale confined $\mathrm{FeRh}$ is present when exploring the magnetic order parameter as well, in addition to electronic order parameters such as resistivity [29]. The recovery of the abrupt transition during cooling may be beneficial for achieving a low-energy switching mechanism between FM and AF phases, where both magnetization and resistivity properties could be jointly utilized. While magnetic, electronic and structural degrees of freedom are in principle commensurate in $\mathrm{FeRh}$ [46], it is important to investigate whether these properties respond simultaneously across the transition at small length scales or very short time scales. 
The dependence of the fit parameters extracted from Eq. (1) on the FeRh structure size is shown in Figure 7. Figure 7(a) displays the transition temperatures $T_{F M-}$ and $T_{A F-F M}$, which stay approximately constant until a width below one micron is reached. Upon further FeRh structure size reduction, a strong monotonic decrease of both $T_{F M-A}$ and $T_{A F-F M}$ is observed. The transition temperatures of the FeRh rectangle with a width of $0.3 \mu \mathrm{m}$ are $15-20 \mathrm{~K}$ lower than for the rectangles with widths larger than one micron, as a result of the previously mentioned strain relaxation process introduced upon patterning. From our systematic study, we see that this effect becomes important upon patterning FeRh into structures with lateral sizes below one micron, as clearly seen in Figure 7(a). The phase transition temperature reduction introduced upon patterning is comparable to the effect of an applied magnetic field as large as $2 \mathrm{~T}[8]$.

In addition, the fitted transition widths $w_{F M-A F}$ and $w_{A F-F M}$ are shown in Figure $7(\mathrm{~b})$. We observe that the temperature span of the transition for most mesoscale FeRh rectangles lies below the transition width of the source film. We also notice that the width of the transition during cooling is, in general, lower than that during heating, even before the transition character clearly changes to abrupt jumps. However, the large error bars in the determined $w_{F M-A F}$ and $w_{A F-F}$ parameters do not allow to establish a clear trend. Finally, Figure 7(c) displays the temperature hysteresis $\Delta T=T_{A F-F M}-T_{F M-A}$ associated with the metamagnetic phase transition. The thermal hysteresis stays within the range of $20-25 \mathrm{~K}$ for the wider FeRh rectangles, while few sub-micron structures feature $\Delta T$ values below $15 \mathrm{~K}$.

\section{Temperature dependence of the coercive field in mesoscale FeRh rectangles}

Apart from the temperature-dependent variation of the Kerr contrast, the hysteresis loop measurements shown in Figure 5 for FeRh mesoscale structures display additional features that can be associated with the occurrence of the metamagnetic phase transition. We have plotted the temperature-dependent coercive field $\mu_{0} H_{C}$ retrieved from the magnetic hysteresis loops for the structures R1 and R0.3 in Figs. 8(a) and 8(b), respectively. We observe that upon cooling, the coercive field increases in both cases. This increase, however, begins at a temperature of about $380 \mathrm{~K}$ in the case of $\mathrm{R} 1$, while coercivity remains constant for the structure R0.3 down to $360 \mathrm{~K}$, increasing its value only below this temperature, where the FM content starts to decrease. During the heating cycle, the coercive field is reduced for increasing 
temperature. The coercive field trends during cooling and heating are shifted in temperature by a value corresponding to the thermal hysteresis of magnetization. In fact, when plotting the coercive field value $\mu_{0} H_{C}$ vs. the corresponding relative FM fraction in the insets of Figures $8(\mathrm{a})$ and $8(\mathrm{~b})$, a correlation between these two quantities is apparent, with the data from the cooling and heating cycles lying on top of each other.

This coercivity increase can be caused by several factors. For instance, it is clear that the temperature variation influences the role of thermally activated processes during magnetization reversal. Upon decreasing the temperature, the probability for magnetization switching to occur at lower applied fields is diminished, such that an increase in the coercive field can be expected. However, this explanation alone cannot account for the two-to-three-fold increase of coercivity across the transition, or the discrepancy in the coercive field during heating and cooling. In addition, the substantially different onset temperature at which the coercive field is modified for the structures R1 and R0.3 clearly suggests that this modification is primarily linked to the occurrence of the phase transition. This is supported by the correlation between the coercive field value and the relative AF (or FM) fraction of the system [see the insets in Figures 8(a) and (b)]. We believe that there are two major reasons for the coercivity increase, which are related to the coexistence of AF and FM domains. On one hand, the presence of intermixed AF and FM phases implies that in order to achieve reversal of the FM phase, applied magnetic field has to be increased in order to balance the loss in Zeeman energy due to the reduction in saturation magnetization. This mechanism can be compared, for instance, to the increase in coercivity that is observed upon approaching the stoichiometric compensation point in sperimagnetic rare-earth transition metal alloys [47]. On the other hand, one also has to take into account the exchange interactions between the AF and FM phases. We can presume that at an AF/FM domain boundary, the FM spins will locally acquire a randomly oriented preferential magnetization axis, as a result of the interaction with the adjacent AF spins. A prominent increase of the coercive field was also reported, for instance, in epitaxial Fe films grown onto single-crystalline $\mathrm{Cu}(100)$, which showed a thickness dependent peak of coervivity associated with the coexistence of ferromagnetic and antiferromagnetic fcc phases of Fe [48]. This contributes to the modification of magnetic anisotropy at the microscale, introducing an effective magnetic disorder into the system [49]. Besides the coercivity increase, the latter argument would also explain why the hysteresis loop shape undergoes a qualitative change through the transition, going from a sharp reversal in the fully FM phase to a smooth, gradual reversal as the FM content decreases (see Figure 5). 


\section{CONCLUSIONS AND OUTLOOK}

In summary, we have investigated the metamagnetic behavior of FeRh films upon mesoscale confinement. We have characterized the temperature-dependent magnetization reversal properties of lithographically patterned FeRh structures with lateral sizes down to $0.3 \mu \mathrm{m}$ using magneto-optical Kerr microscopy. We have focused primarily on rectangular FeRh structures due to the possibility of reducing one lateral dimension to the sub-micron scale while still keeping a good signal-to-noise ratio. Another advantage is the conceptually simpler magnetization reversal of elongated structures, featuring shape-induced uniaxial magnetic anisotropy. Following the magnetization vs. temperature dependence of individual FeRh structures across the transition, the transition temperature of individual structures was determined. The systematic study revealed that confining epitaxial FeRh films into lateral sizes below one micron brings a substantial decrease of the transition temperature by about 15-20 K. We argue that this is a result of the strain relaxation process introduced upon patterning, given the increased surface-to-volume ratio of the mesoscale structure. We have also observed that for confinement lengths below $0.5 \mu \mathrm{m}$, the transition becomes very sharp during the cooling cycle, as a result of the different role of AF and FM order in the presence of disorder such as crystallographic defects. This recovery of the abrupt character of the first-order transition and the suppression of phase separation could lead to efficient routes to control the AF or FM order in FeRh-based devices with low-energy external driving forces. For instance, the combination of shape anisotropy as well as the strain-control of the out-of-plane magnetocrystalline anisotropy [39] could constitute useful ingredients for achieving novel and efficient device functionalities based on the metamagnetic behavior of FeRh. While in principle Kerr microscopy allows spatially resolved imaging of the AF and FM phases, the optical resolution limit only permits to gain coarse details of processes such as domain nucleation and growth. For a comprehensive AF/FM domain study across the first-order phase transition, scanning probe techniques [50] or x-ray microscopy [27] may prove useful to gain further insight on confinement effects in the metamagnetic behavior of FeRh. 


\section{Acknowledgments:}

We acknowledge the Grant Agency of the Czech Republic (grant no. 16-23940Y) and the Ministry of Education, Youth and Sports of the Czech Republic under the projects CEITEC 2020 (LQ1601) and CEITEC Nano (LM2015041). E.F. acknowledges the U.S. Department of Energy, Office of Basic Energy Sciences award \#DE-SC0003678 supporting Kerr microscopy measurements. This work has received funding from the European Union's Horizon 2020 research and innovation programme under the Marie Skłodowska-Curie and it is co-financed by the South Moravian Region under grant agreement No. 665860.

\section{$\underline{\text { References: }}$}

[1] M. Fallot, and R. Hocart, "Sur l'apparition du ferromagnétisme par élévation de température dans des alliages de fer et de rhodium", Rev. Sci. 77, 498 (1939)

[2] G. Shirane, R. Nathans and C. W. Chen, "Magnetic moments and unpaired spin densities in the Fe-Rh alloys", Phys. Rev. 134, A1547 (1964).

[3] L. J. Swartzendruber, “The Fe-Rh (Iron-Rhodium) System”, Bull. Alloy Phase Diagr. 5 $456(1984)$

[4] G. Shirane, C. W. Chen, P. A. Flinn, and R. Nathans, "Hyperfine Fields and Magnetic Moments in the Fe-Rh System", J. Appl. Phys. 34, 1044 (1963)

[5] V. L. Moruzzi and P. M. Marcus, "Antiferromagnetic-ferromagnetic transition in FeRh", Phys. Rev. B 46, 2864 (1992)

[6] J. S. Kouvel, and C. C. Hartelius, “Anomalous Magnetic Moments and Transformations in the Ordered Alloy FeRh", J. Appl. Phys. 33, 1343 (1962)

[7] L. H. Lewis, C. H. Marrows, and S. Langridge, "Coupled magnetic, structural and electronic phase transitions in FeRh", J. Phys. D: Appl. Phys. 49, 323002 (2016)

[8] S. Maat, J.-U. Thiele, and E. E. Fullerton, "Temperature and field hysteresis of the antiferromagnetic-to-ferromagnetic phase transition in epitaxial FeRh films", Phys. Rev. B 72, $214432(2005)$

[9] R. O. Cherifi et al., "Electric-field control of magnetic order above room temperature", Nat. Mater. 13, 345 (2014).

[10] S. P. Bennett et al., "Giant Controllable Magnetization Changes Induced by Structural Phase Transitions in a Metamagnetic Artificial Multiferroic”, Sci. Rep. 6, 22708 (2016) 
[11] Q. B. Hu, J. Li, C. C. Wang, Z. J. Zhou, Q. Q. Cao, T. J. Zhou, D. H. Wang, and Y. W. $\mathrm{Du}$, "Electric field tuning of magnetocaloric effect in $\mathrm{FeRh}_{0.96} \mathrm{Pd}_{0.04} / \mathrm{PMN}-\mathrm{PT}$ composite near room temperature", Appl. Phys. Lett. 110, 222408 (2017)

[12] N. Matsuzaki, T. Moriyama, M. Nagata, K.-J. Kim, I. Suzuki, T. Taniyama, and T. Ono, "Current induced antiferro-ferromagnetic transition in FeRh nanowires", Jpn. J. Appl. Phys. 54, 073002 (2015)

[13] T. Moriyama, N. Matsuzaki, K.-J. Kim, I. Suzuki, T. Taniyama, and T. Ono, "Sequential write-read operations in FeRh antiferromagnetic memory", Appl. Phys. Lett. 107, 122403 (2015)

[14] I. Suzuki, T. Naito, M. Itoh, and T. Taniyama, "Barkhausen-like antiferromagnetic to ferromagnetic phase transition driven by spin polarized current", Appl. Phys. Lett. 107, 082408 (2015)

[15] J.-U. Thiele, M. Buess, and C. H. Back, "Spin dynamics of the antiferromagnetic-toferromagnetic phase transition in FeRh on a sub-picosecond time scale", Appl. Phys. Lett. 85, 2857 (2004)

[16] G. Ju, J. Hohlfeld, B. Bergman, R. J.M. van deVeerdonk, O. N. Mryasov, J.-Y. Kim, X. $\mathrm{Wu}, \mathrm{D}$. Weller, and B. Koopmans, "Ultrafast Generation of Ferromagnetic Order via a LaserInduced Phase Transformation in FeRh Thin Films”, Phys. Rev. Lett. 93, 197403 (2004)

[17] S. O. Mariager et al., "Structural and Magnetic Dynamics of a Laser Induced Phase Transition in FeRh", Phys. Rev. Lett. 108, 087201 (2012)

[18] J.-U. Thiele, S. Maat, and E.E. Fullerton, "FeRh/FePt exchange spring films for thermally assisted magnetic recording media”, Appl. Phys. Lett. 82, 2859 (2003)

[19] X. Martí et al., "Room-temperature antiferromagnetic memory resistor", Nat. Mater. 13, 367 (2014)

[20] M. P. Annaorazov, K. A. Asatryan, G. Myalikgulyev, S. A. Nikitin, A.M.Tishin, A. L. Tyurin, "Alloys of the Fe-Rh system as a new class of working material for magnetic refrigerators", Cryogenics 32, 867 (1992)

[21] E. Stern-Taulats, A. Planes, P. Lloveras, M. Barrio, J.-L. Tamarit, S. Pramanick, S. Majumdar, C. Frontera, and L. Mañosa, "Barocaloric and magnetocaloric effects in $\mathrm{Fe}_{49} \mathrm{Rh}_{51}$ ", Phys. Rev. B 89, 214105 (2014)

[22] S. B. Roy, G. K. Perkins, M. K. Chattopadhyay, A. K. Nigam, K. J. S. Sokhey, P. Chaddah, A. D. Caplin, and L. F. Cohen, "First Order Magnetic Transition in Doped CeFe2 Alloys: Phase Coexistence and Metastability”, Phys. Rev. Lett. 92, 147203 (2004)

[23] Y. Ohtani, and I. Hatakeyama, "Antiferro-ferromagnetic transition and microstructural properties in a sputter deposited FeRh thin film system", J. Appl. Phys. 74, 3328 (1993) 
[24] Y. Ohtani, and I. Hatakeyama, "Features of broad magnetic transition in FeRh thin film", J. Magn. Magn. Mater. 131, 339 (1994)

[25] M. Loving, F. Jimenez-Villacorta, B. Kaeswurm, D. A. Arena, C. H. Marrows, and L. H. Lewis, "Structural evidence for stabilized ferromagnetism in epitaxial FeRh nanoislands", $J$. Phys. D: Appl. Phys. 46, 162002 (2013)

[26] M. A. de Vries et al., "Asymmetric 'melting' and 'freezing' kinetics of the magnetostructural phase transition in B2-ordered FeRh epilayers", Appl. Phys. Lett. 104, 232407 (2014)

[27] C. Baldasseroni, C. Bordel, C. Antonakos, A. Scholl, K. H. Stone, J. B. Kortright, and F. Hellman, "Temperature-driven growth of antiferromagnetic domains in thin-film FeRh", $J$. Phys.: Condens. Matter 27, 256001 (2015)

[28] L. Lounis et al., "Temperature and field dependent magnetization in a sub-m patterned Co/FeRh film studied by resonant x-ray scattering”, J. Phys. D: Appl. Phys. 49, 205003 (2016)

[29] V. Uhlír, J. A. Arregi, and E. E. Fullerton, "Colossal magnetic phase transition asymmetry in mesoscale FeRh stripes”, Nat. Commun. 7, 13113 (2016)

[30] M. R. Fitzsimmons et al., “Antiferromagnetic domain size and exchange bias”, Phys. Rev. $B$ 77, 224406 (2008)

[31] E. Nikulina, O. Idigoras. P. Vavassori, A. Chuvilin, and A. Berger, "Magneto-optical magnetometry of individual $30 \mathrm{~nm}$ cobalt nanowires grown by electron beam induced deposition”, Appl. Phys. Lett. 100, 142401 (2012)

[32] J. McCord, "Progress in magnetic domain observation by advanced magneto-optical microscopy", J. Phys. D: Appl. Phys. 48, 333001 (2015)

[33] R. P. Cowburn, D. K. Koltsov, A. O. Adeyeye, and M. E. Welland, "Probing submicron nanomagnets by magneto-optics", Appl. Phys. Lett. 73, 3947 (1998)

[34] D. A. Allwood, G. Xiong, M. D. Cooke, and R. P. Cowburn, "Magneto-optical Kerr effect analysis of magnetic nanostructures", J. Phys. D: Appl. Phys. 36, 2175 (2003)

[35] L. Flajšman, M. Urbánek, V. Křižáková, M. Vaňatka, I. Turčan, and T. Šikola, "Highresolution fully vectorial scanning Kerr magnetometer”, Rev. Sci. Instrum. 87, 053704 (2016)

[36] V. Saidl et al., "Investigation of magneto-structural transition in FeRh by reflectivity and transmittance measurements in visible and near-infrared spectral region", New. J. Phys. 18, 083017 (2016)

[37] C. Le Graët et al., "Temperature controlled motion of an antiferromagnet- ferromagnet interface within a dopant-graded FeRh epilayer”, APL Materials 3, 041802 (2015)

[38] D. Sander et al., “The 2017 Magnetism Roadmap”, J. Phys. D: Appl. Phys. 50, 363001 (2017) 
[39] C. Bordel et al., "Fe Spin Reorientation across the Metamagnetic Transition in Strained FeRh Thin Films", Phys. Rev. Lett. 109, 117201 (2012)

[40] F. Pressacco, V. Uhlír̆, M. Gatti, A. Bendounan, E. E. Fullerton, and F. Sirotti, "Stable room-temperature ferromagnetic phase at the FeRh(100) surface", Sci. Rep. 6, 22383 (2016)

[41] M. Jiang, X. Z. Chen, X. J. Zhou, Y. Y. Wang, F. Pan, and C. Song, "Influence of film composition on the transition temperature of FeRh films", J. Cryst. Growth 438, 19 (2016)

[42] T. Thomson, G. Hu, and, B. D. Terris, "Intrinsic distribution of magnetic anisotropy in thin films probed by patterned nanostructures”, Phys. Rev. Lett. 96, 257204 (2006)

[43] T. Sebastian, A. Conca, G. Wolf, H. Schultheiss, B. Leven, and B. Hillebrands, "Magnetooptical investigation of the shape anisotropy of individual micron-sized magnetic elements", $J$. Appl. Phys. 110, 083909 (2011)

[44] J. A. Johnson, M. Grimsditch, V. Metlushko, P. Vavassori, B. Ilic, P. Neuzil and R. Kumar, "Magneto-optic Kerr effect investigation of cobalt and permalloy nanoscale dot arrays: Shape effects on magnetization reversal", Appl. Phys. Lett. 77, 4410 (2000)

[45] F. Radu et al., "Origin of the reduced exchange bias in an epitaxial FeNi(111)/CoO(111) bilayer", Phys. Rev. B 79, 184425 (2009)

[46] J.-U. Thiele, S. Maat, J. L. Robertson, and E. E. Fullerton, "Magnetic and Structural Properties of FePt-FeRh Exchange Spring Films for Thermally Assisted Magnetic Recording Media", IEEE Trans. Magn. 40, 2537 (2004)

[47] M. Gottwald, M. Hehn, F. Montaigne, D. Lacour, G. Lengaigne, S. Suire, and S. Mangin, "Magnetoresistive effects in perpendicularly magnetized Tb-Co alloy based thin films and spin valves", J. Appl. Phys. 111, 083904 (2012)

[48] A. Berger, B. Feldmann, H. Zillgen, and M. Wuttig, "Correlation between the microscopic and macroscopic magnetic properties in ultrathin $\mathrm{Fe} / \mathrm{Cu}(100)$-films", J. Magn. Magn. Mater. 183, 35 (1998)

[49] A. Berger, A. Inomata, J. S. Jiang, J. E. Pearson, and S. D. Bader, "Experimental Observation of Disorder-Driven Hysteresis-Loop Criticality”, Phys. Rev. Lett. 85, 4176 (2000) [50] J. Shao et al., "Emerging single-phase state in small manganite nanodisks", Proc. Natl. Acad. Sci. U.S.A. 113, 9228 (2016) 


\section{Figure 1}

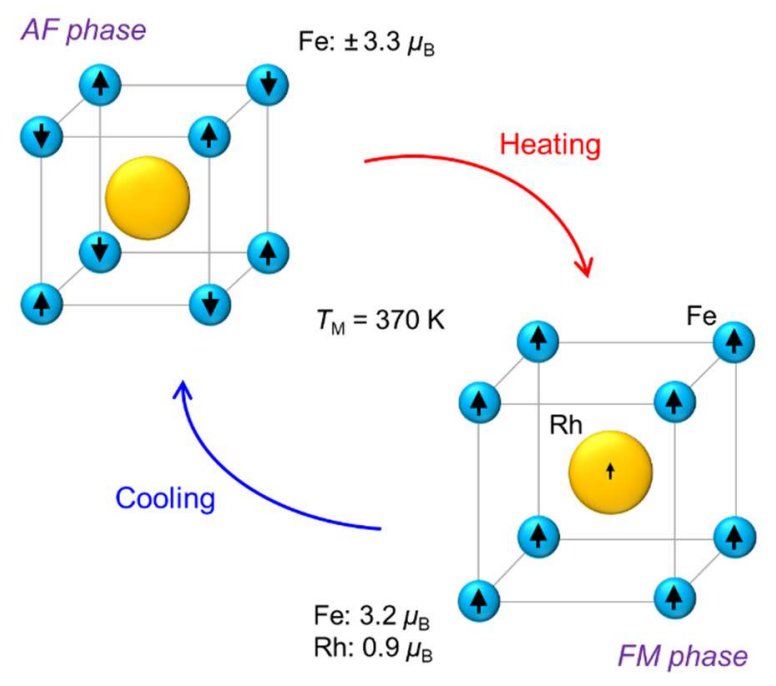

Figure 1: Schematic representation of the structure and magnetic moment configuration across the metamagnetic phase transition in FeRh. In the FM phase, both $\mathrm{Fe}$ and $\mathrm{Rh}$ have ferromagnetically coupled ordered moments, whereas in the AF phase only the Fe atoms display magnetic moments that are antiferromagnetically coupled with their nearest neighbors (G-type antiferromagnetism). The magnetic moments per atom are indicated for each phase. 
Figure 2
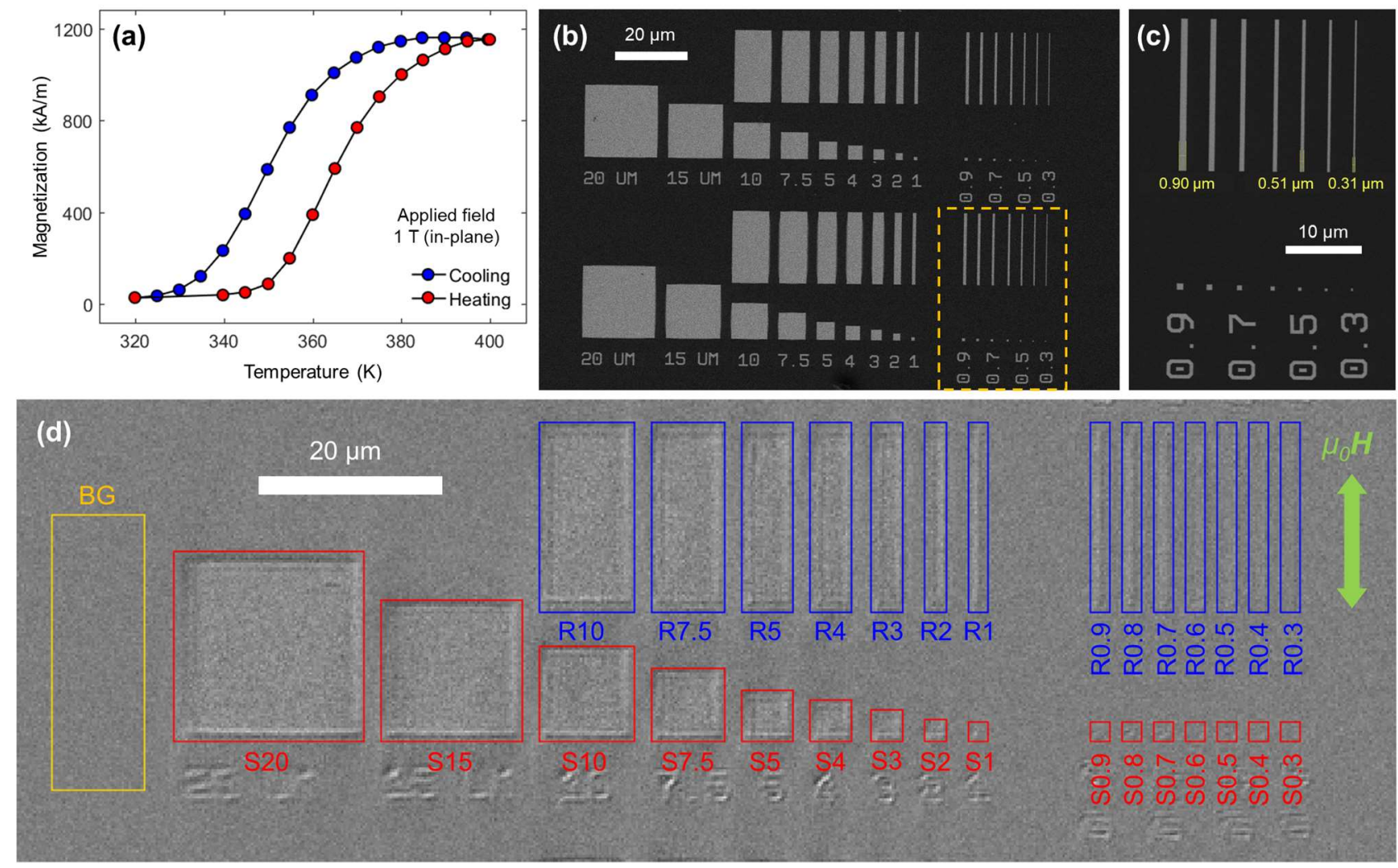

Figure 2: (a) Magnetization vs. temperature for the 50-nm-thick source film patterned into the mesoscale FeRh structures, measured under an in-plane applied field of 1 T. (b) Scanning electron micrograph of an array of mesoscale FeRh rectangles and squares with lateral sizes between 0.3 and $20 \mu \mathrm{m}$. The area limited by the dashed lines in (b) is shown under a larger magnification in (c), which exhibits well-defined $20-\mu \mathrm{m}$-long stripes as well as square structures with sizes between 0.3 and $0.9 \mu \mathrm{m}$. (d) Kerr microscopy image ( $T=400 \mathrm{~K}, \mu_{0} H=$ $40 \mathrm{mT}$ ) over an area of $150 \times 50 \mu \mathrm{m}^{2}$ of the same FeRh squares and rectangles shown in (b). Individual structures are delimited by a rectangular region of interest (ROI), within which the longitudinal Kerr contrast during magnetization reversal is evaluated. The green arrow in the right indicates the direction of the applied magnetic field, defined along the long axis of the rectangular structures. The ROIs for squares and rectangles are depicted as red and blue, respectively. In the left part of the image, a yellow rectangle of an area of $15 \times 40 \mu \mathrm{m}^{2}$ is defined on the $\mathrm{MgO}$ substrate at the etched part of the film, in order to obtain the background (BG) signal. 


\section{Figure 3}

(a)
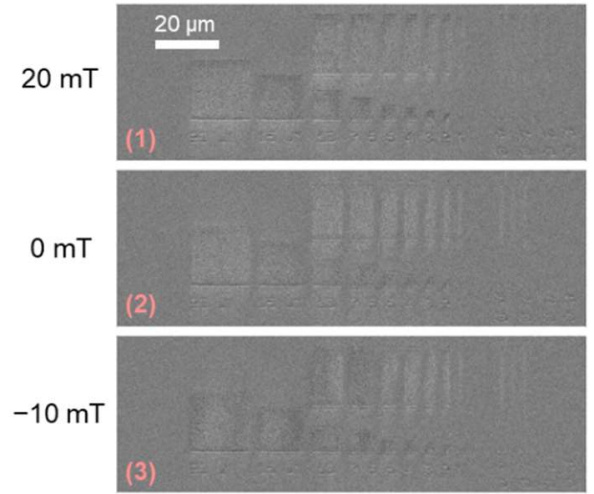

$-12 \mathrm{mT}$

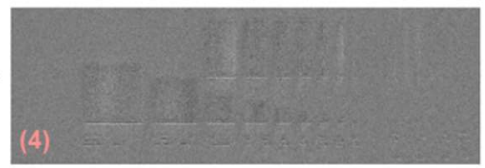

$-20 \mathrm{mT}$

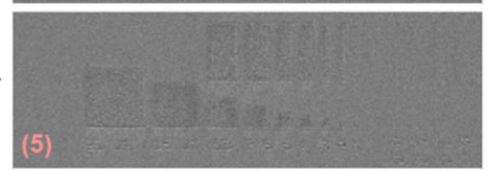

Increasing field branch $(\triangleright)$

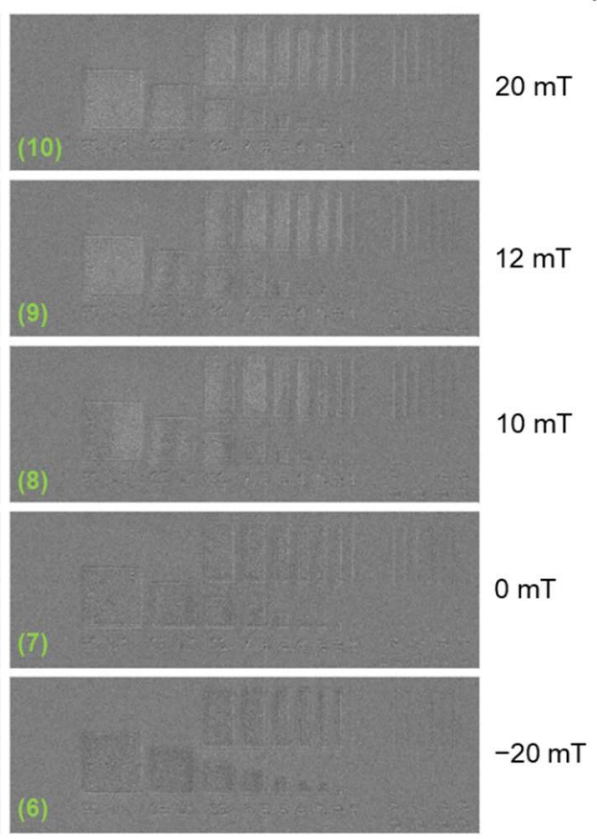

(b)

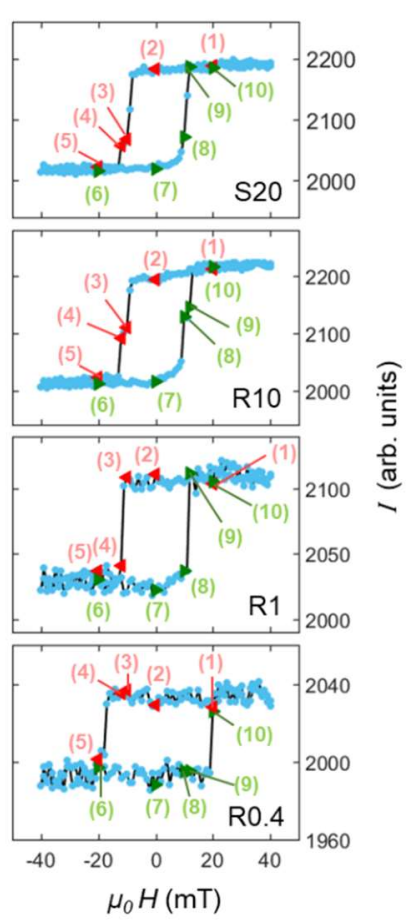

Figure 3: (a) Kerr microscopy snapshots of mesoscale FeRh squares and rectangles acquired at $400 \mathrm{~K}$ and different applied magnetic field values during magnetization reversal, both for decreasing (left panel) and increasing (right panel) field branches. The grayscale represents the magnetization component along the vertical direction. (b) Magnetization hysteresis loops for the FeRh structures S20, R10, R1 and R0.4, obtained by evaluating the grayscale signal $I$ variation within their respective region of interest. The field values corresponding to the snapshots in (a) are indicated in the hysteresis loops by the snapshot number as well as by leftpointing or right-pointing triangle markers (for decreasing or increasing field branches, respectively). 


\section{Figure 4}

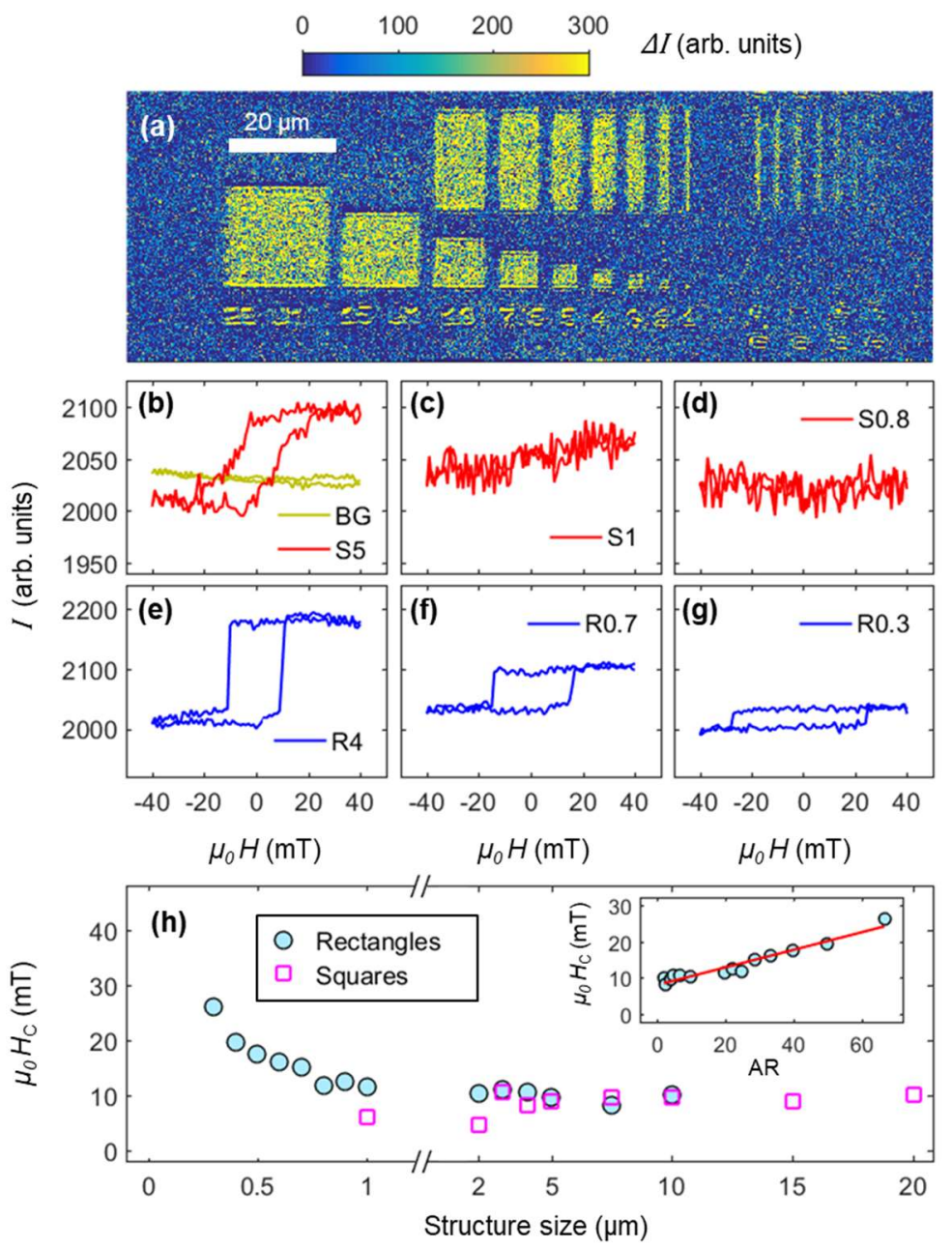

Figure 4: (a) Magnetic domain contrast at $400 \mathrm{~K}$ obtained by comparing Kerr micrographs at opposite applied magnetic fields of $\pm 40 \mathrm{mT}$. Below, magnetic hysteresis loops at $400 \mathrm{~K}$ for the FeRh squares (b) S5, (c) S1 and (d) S0.8, as well as for the rectangles (e) R4, (f) R0.7 and (g) R0.3. The field-dependent Kerr signal $I$ arising from the background is plotted in (b) for reference. (h) Experimentally determined coercive field values $\mu_{0} H_{C}$ at $400 \mathrm{~K}$ vs. the characteristic size of rectangular and square FeRh structures. The inset shows $\mu_{0} H_{C}$ dependence on the aspect ratio (AR) of the rectangular structures. The red solid line is a linear fit to the experimental data. 


\section{Figure 5}
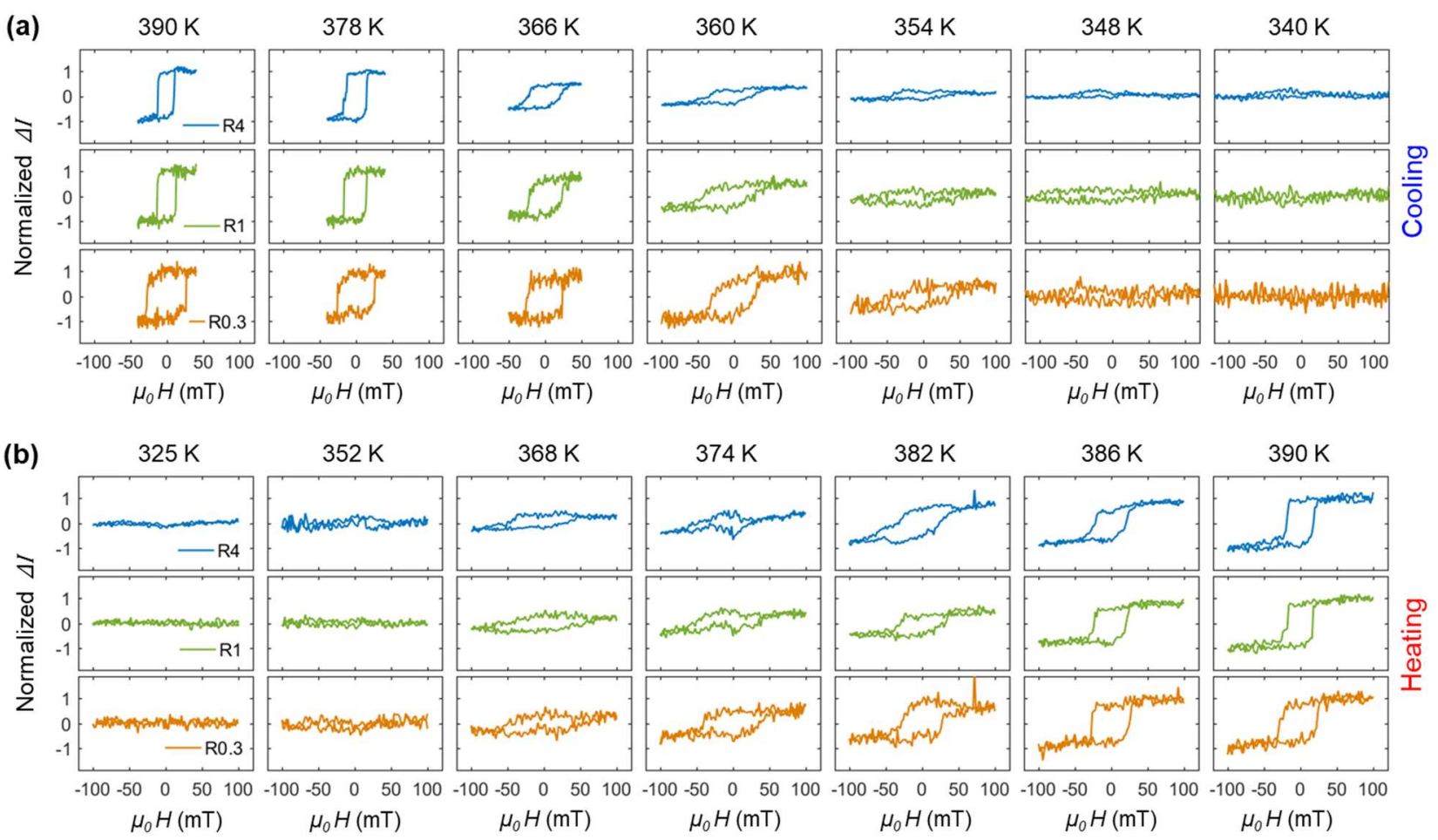

Figure 5: Temperature-dependent magnetic hysteresis loops measured for the FeRh rectangles R4, R1 and R0.3 at selected temperatures during the (a) cooling and (b) heating thermal cycles. For each structure investigated, the relative change in the Kerr signal $\Delta I$ is scaled to the normalized Kerr signal level of the magnetization saturation at $390 \mathrm{~K}$. 


\section{Figure 6}
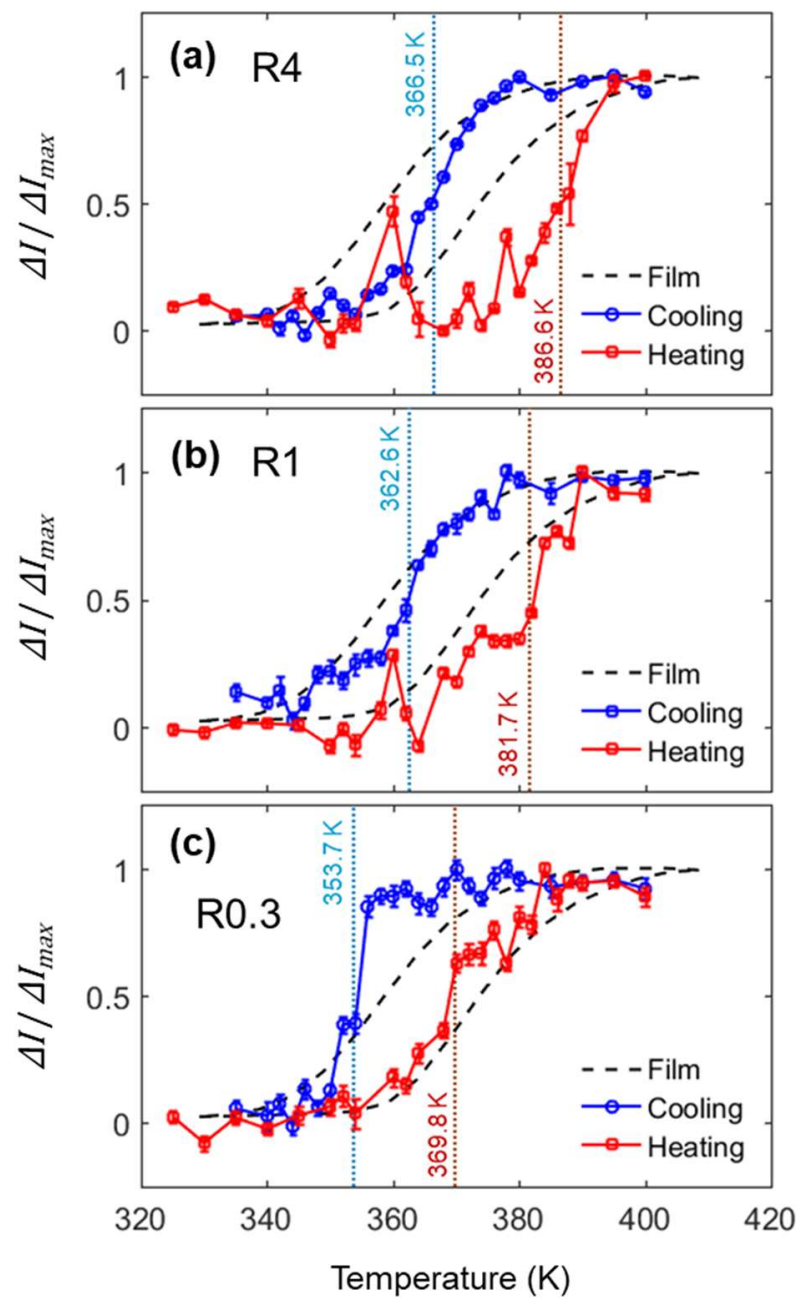

Figure 6: Temperature-dependent variations of the Kerr contrast in magnetic saturation for individual $20-\mu \mathrm{m}$-long FeRh rectangles with widths of (a) $4 \mu \mathrm{m}$, (b) $1 \mu \mathrm{m}$ and (c) $0.3 \mu \mathrm{m}$. Data are shown for both the cooling (blue circles) and heating (red squares) cycles across the phase transition. The vibrating sample magnetometry data of the original FeRh film were normalized and corrected for the temperature offset $-8 \mathrm{~K} / \mathrm{T}$ due to the applied magnetic field (indicated by the dashed line). The vertical dotted lines indicate the transition temperatures obtained by fitting the data to Eq. (1). 
Figure 7

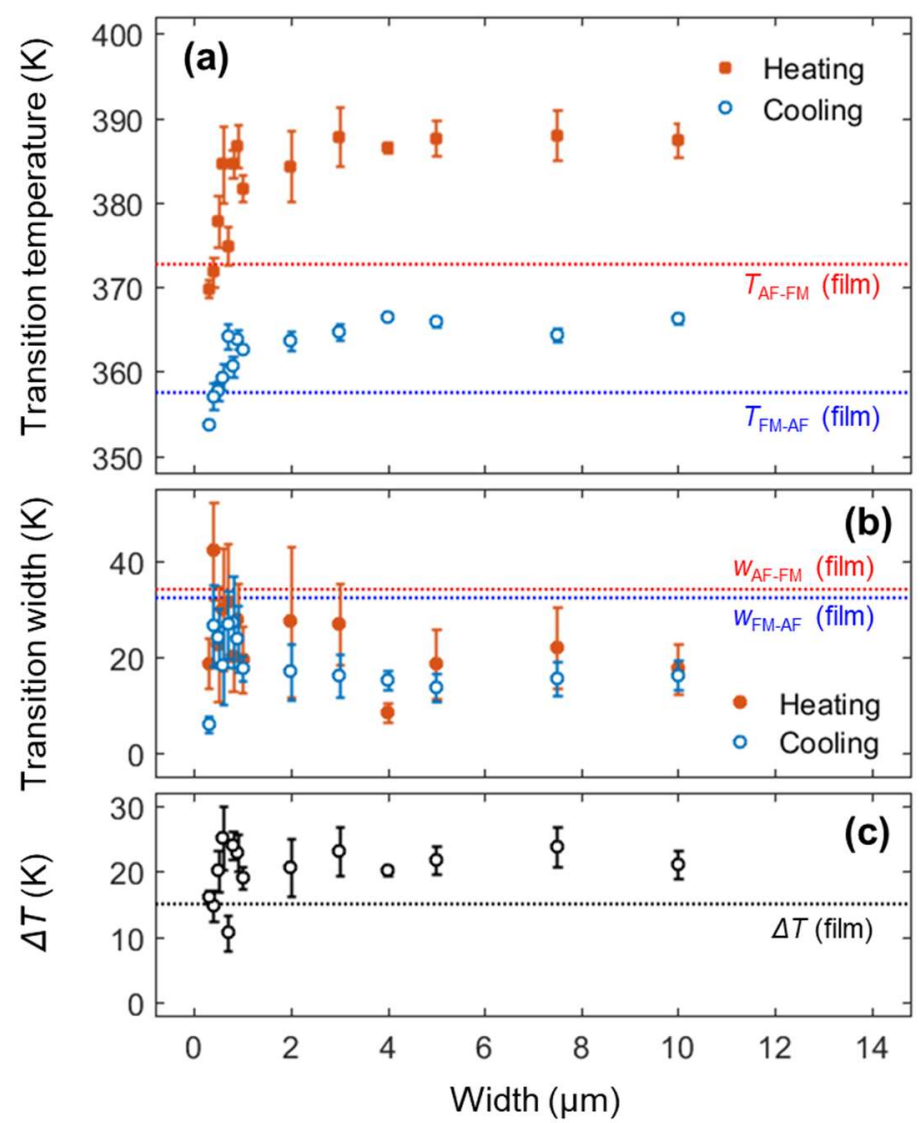

Figure 7: (a) Metamagnetic phase transition temperatures $T_{F M-A F}, T_{A F-F M}$, (b) transition widths $w_{F M-A F}, w_{A F-F M}$ and (c) temperature hysteresis $\Delta T$ for individual mesoscale FeRh rectangles with widths in the range of $0.3-10 \mu \mathrm{m}$. The horizontal dotted lines indicate the phase transition features of the source FeRh film, as extracted from vibrating sample magnetometry data. 


\section{Figure 8}
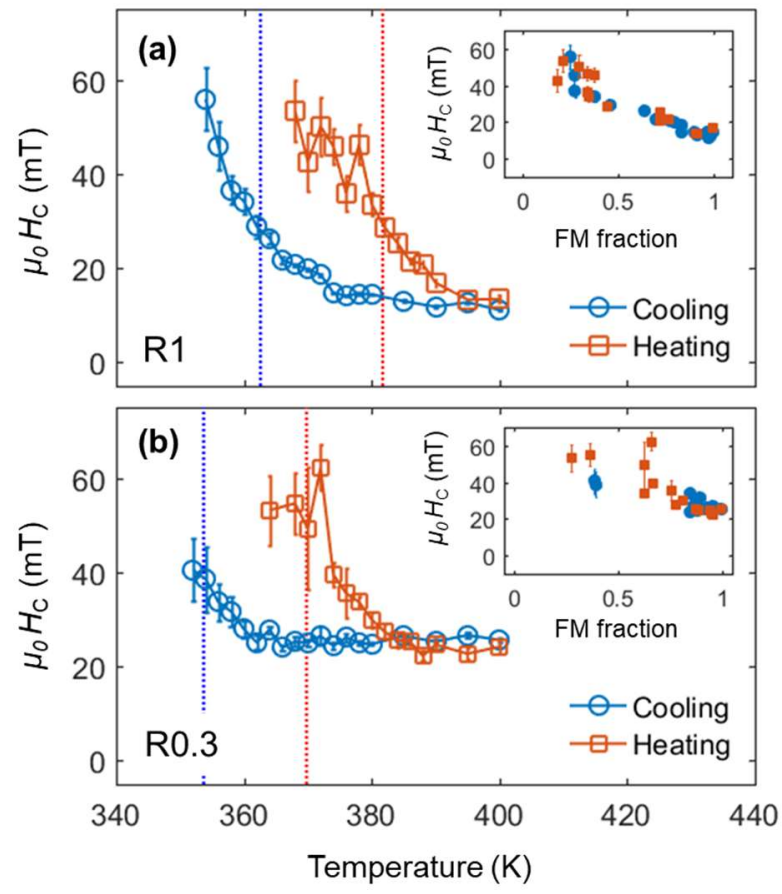

Figure 8: Temperature dependence of the coercive field $\mu_{0} H_{C}$ for the rectangular FeRh structures (a) R1 and (b) R0.3, during the heating and cooling cycles. The inset in (a) and (b) show $\mu_{0} H_{C}$ vs. $\Delta I / \Delta I_{\text {max }}$ (or the relative FM fraction) during heating and cooling. The vertical dotted lines indicate the FM-AF and AF-FM transition temperatures as extracted from Eq. (1). 